\title{
Zwischen rinks und lechts. Angstpolitik in Zei- ten des Populismus
}

Veith Selk

\section{Einleitung: Angstpolitik in der politischen Gesell- schaft}

Unsere Gesellschaft ist eine "politische Gesellschaft«. ${ }^{1}$ In ihr können alle Institutionen und Handlungen politisiert werden. Die dem Feminismus entstammende Parole "Das Private ist politisch" bringt diese Erfahrung zum Ausdruck. Im Sinne dieses Slogans haben die Protestbewegungen der Siebzigerjahre und die "partizipative Revolution « ${ }^{2}$ der Achtzigerjahre zu erweiterten Beteiligungschancen und einem umfassenden Politisierungsprozess geführt. In den von der politischen Klasse dominierten Parteiendemokratien ${ }^{3}$ ging Politisierung vor allem vom Staatsapparat aus, in der politisierten Demokratie der Gegenwart wird sie auch von der Bürgerschaft vorangetrieben. ${ }^{4}$

Der US-amerikanische Philosoph John Dewey hatte bereits am Anfang des 20. Jahrhunderts beobachtet, dass in der modernen Gesellschaft mehr und mehr Bürger von den Folgen des Handelns ihrer Mitbürger betroffen sind, weshalb jene ein Interesse an der Regulierung dieser Folgen haben. ${ }^{5}$ Er schlussfolgerte daraus, dass in der Demokratie der Bereich des als regulierungsbedürftig Angesehenen

\footnotetext{
1 Michael Th. Greven, Die politische Gesellschaft. Kontingenz und Dezision als Probleme des Regierens und der Demokratie, Wiesbaden ${ }^{2} 2009$.

2 Max Kaase, The challenge of the "participatory revolution" in pluralist democracies, in: International Political Science Review 5 (1984), 299-318; Russel J. Dalton, Citizenship Norms and the Expansion of Political Participation, in: Political Studies 56 (2008), 76-98.

3 Klaus von Beyme, Die politische Klasse im Parteienstaat, Frankfurt am Main 1993; Gerhard Leibholz, Strukturprobleme der modernen Demokratie, Karlsruhe 1958.

4 Politisierung geht damit auch von der nicht zur politischen Elite gehörenden Mittelklasse und deren "Gelegenheitspolitikern" (Max Weber) aus. Diejenigen, die sich im sozialstrukturellen Sinne "unter« der Mittelklasse befinden, partizipieren hingegen weniger an Politik; siehe Armin Schäfer, Der Verlust politischer Gleichheit. Warum ungleiche Beteiligung der Demokratie schadet, in: Staatstätigkeiten, Parteien und Demokratie, hg. v. Klaus Armingeon, Wiesbaden 2013, 547-566.

5 John Dewey, The Public and its Problems, New York 1927.
} 
wächst und die Öffentlichkeit immer mehr Handlungen umfasst. Das Politische berührt nun Bereiche, die vormals als unpolitisch und privat galten.

Die beteiligungsorientierte Linke der zweiten Hälfte des letzten Jahrhunderts bewertete diesen Politisierungsprozess positiv. Sie sah in ihm die Möglichkeit angelegt, Partizipationsmöglichkeiten auszuweiten und die Demokratisierung der Gesellschaft voranzutreiben, ${ }^{6}$ während die etatistische Rechte ihn als eine Bedrohung bewährter Institutionen beurteilte. ${ }^{7}$ Heute hingegen scheinen vor allem Populistinnen und ihre Anhänger von der Politisierung der Gesellschaft eine nachhaltige Demokratisierung zu erwarten; die Antipopulisten aus allen Lagern wiederum deuten Politisierung als ein Problem, das zu mehr Polarisierung führe, die Europäische Union gefährde und die Stabilität der liberalen Demokratien bedrohe.

Die rasch voranschreitende Digitalisierung der Kommunikationstechnologie beschleunigt den Prozess der Politisierung. Sie weitet die Beteiligung an politischer Kommunikation im Kontext digitaler Politikformen aus. Das ist folgenreich, weil die politisch relevante Kommunikation von den politischen Eliten nun nicht mehr so leicht kontrolliert, gefiltert oder auch nur antizipiert werden kann. ${ }^{8}$ Deren Mitglieder in Parteien, Wirtschaft und Journalismus scheinen zudem überrascht darüber zu sein, was für Meinungen über sie »im Volk« kursieren, die nun durch die Öffnung politischer Kommunikation zu Tage befördert werden.

Führt die kommunikativ »offene Gesellschaft» damit zur Wiederkehr der Angst der Eliten vor der populistisch gestimmten Masse? Gerade die politisch sichtbaren Mitglieder der Eliten haben durchaus Grund zur Angst, denn aus Mordphantasien im Netz wurden bereits Anschläge im wirklichen Leben. Einige derjenigen, die sich in Fundamentalopposition zu den »korrupten Eliten « sehen, würden dies womöglich damit rechtfertigen, dass sich die happy few den Volkszorn nicht ohne Grund zuzögen. In diesem Kontext wirkt die Hoffnung auf eine Verbesserung der öffentlichen Debatten durch digitale und allseitig vernetzte Kommunikation im Internet illusorisch. Zudem entsteht der Eindruck, dass »die da oben" auf den Kommandohöhen von Politik, Wirtschaft und Kultur zunehmend

\footnotetext{
6 Fritz Vilmar, Strategien der Demokratisierung, 2 Bände, Darmstadt 1974.

7 Wilhelm Hennis, Demokratisierung. Zur Problematik eines Begriffs, Köln/Opladen 1970.

8 Stephen Coleman/Deen Freelon, Introduction: conceptualizing digital politics, in: Handbook of digital politics, hg. v. Stephen Coleman/Deen Freelon, London 2015, $1-13$.
} 
Unbehagen gegenüber den Bürgerinnen und Bürgern verspüren, während »die da unten« sowohl den Eliten als auch ihren Mitbürgerinnen und Mitbürgern nicht über den Weg trauen. Hinzu kommen die um sich greifende Krisensemantik und die sich eintrübenden Aussichten auf eine Verbesserung der westlichen Demokratien. ${ }^{9}$ In Anbetracht dieser Lage kann man Angst eine steile politische Karriere vorausagen.

In dieser Konstellation erfährt ein Politikmodus eine Renaissance, den ich im Folgenden als Angstpolitik bezeichnen will. Meine These ist, dass er insbesondere im Rechtspopulismus wirkmächtig ist. Er tritt dort als Angstpolitik auf, die zwischen links und rechts changiert. Die Antipopulisten in Parteien und Gesellschaft reagieren hierauf mit einer anderen Form von Angstpolitik. In ihr kehrt der Topos der Angst vor der irrationalen Masse wieder.

Angstpolitik zeichnet sich dadurch aus, dass in ihr politische Forderungen mit Angst begründet werden und affirmativ auf Angst Bezug genommen wird, zum Beispiel auf die Angst vor der Klimakatastrophe, die Angst vor muslimischen Migranten oder die Angst vor dem wirtschaftlichen Abstieg. Angstpolitik unterscheidet sich damit von zwei anderen Typen politischen Handelns, Identitätspolitik und Interessenpolitik. Identitätspolitik ist das Politikmachen mit Bezug auf Identitäten, während in der Interessenpolitik mit Interessen Politik gemacht wird. Wer hingegen Angstpolitik treibt, der setzt Angst an den Anfang. ${ }^{10}$

Interessenpolitik folgt dem principal-agent-Model. Politikerinnen und Interessenvertreter nehmen gesellschaftlich relevant gewordene Interessen auf, organisieren sie und bringen sie im politischen Aushandlungskonflikt zur Geltung. Das Ergebnis ist in der Regel ein Kompromiss, der von den Prinzipalen bilanziert werden kann. Im Unterschied dazu besteht Identitätspolitik aus der Repräsentation von Identitäten (zumeist der Identität des "großen Ganzen" oder der Identität von Gruppen). Sie kann von den jeweiligen Adressaten zwar dahingehend bewertet werden, ob diese sich in der repräsentativen Inszenierung wiederfinden, aber im Unterschied zur Interessenpolitik ist die Repräsentation von Identität symbolisch, sie ist deshalb nicht zweckrational bilanzierbar und insoweit ideologieanfälliger. ${ }^{11}$

9 David Runciman, How Democracy Ends, London 2019.

${ }^{10}$ Oder fordert diese ein, so wie Greta Thunberg mit ihrem Aufruf "I want you to panic!

${ }^{11}$ Diese Definition von Interessen- und Identitätspolitik folgt Heinz Steinert, Kulturindustrielle Politik mit dem Großen \& Ganzen: Populismus, Politik-Darsteller, ihr Publikum und seine Mobilisierung, in: IPG 4 (1999), 402-413. Die idealtypische Unterscheidung darf natürlich nicht darüber hinwegtäuschen, dass diese Politikfor- 
Angstpolitik weist in dieser Hinsicht eine gewisse Nähe zur Identitätspolitik auf, denn sie bezieht sich prognostisch auf mögliche $\mathrm{Ri}$ siken in der Zukunft, die, wenn sie abstrakt formuliert sind, schwer zu widerlegen sind. In der Angstpolitik werden politische Entscheidungen mit Rekurs auf Angst vor einem möglichen, hinreichend wahrscheinlich erscheinenden Übel gerechtfertigt, das abgewehrt werden soll. Ihr Ausgangspunkt ist die Prognose einer drohenden Gefährdung durch kommende Ereignisse und Entwicklungen oder die Behauptung einer akuten Bedrohung durch das Handeln von "Feinden" oder "Gegnern". Eine gesellschaftlich relevante Angst wird hierbei als ein Indikator für die Korrektheit der Prognose dargestellt und performativ verstärkt.

Angstpolitik ist Politik für Krisenzeiten. Sie kann von ihren Protagonisten dann besonders authentisch auf die politische Bühne gebracht werden. Die Möglichkeit, Angstpolitik zu treiben ist zugleich strukturell in unserer Gesellschaft angelegt. In der Angstkultur der "flüssigen Moderne ${ }^{12}$ ist sie immer möglich. Auf die entfremdete und stabilisierte Ordnung der "spätkapitalistischen Industriegesellschaft ${ }^{13}$ folgte der neoliberale Beunruhigungskapitalismus. In ihm ist Angst endemisch. Angstpolitik ist deshalb nicht auf Situationen angewiesen, in denen sich kurzfristig Gelegenheitsfenster für Mobilisierung öffnen. Hinzu kommt, dass die liberale Demokratie für Angstpolitik empfänglich ist. Mit ihrer nur schwach restringierten Öffentlichkeit institutionalisiert sie eine Sphäre flottierender Angstkommunikation, die sich auf nahezu alle möglichen Risiken beziehen kann. Sie erlangt leicht eine politische Bedeutung, weil Angst in der liberalen Öffentlichkeit als ein politisches Argument und als normativer Aufmerksamkeitsmarker fungiert. ${ }^{14}$ Wer in der Öffentlichkeit Angst artikuliert, der signalisiert damit, die andere Seite müsse nun erst einmal zuhören. ${ }^{15}$

men in der politischen Praxis fast nie rein auftreten, sondern in unterschiedlicher Mischung miteinander verbunden werden. Das gilt auch für Angstpolitik.

12 Zygmunt Bauman, Liquid Fear, Cambridge 2006.

${ }^{13}$ Deren Stabilität in den Sechziger Jahren sowohl von links als auch von rechts betont worden ist; siehe Herbert Marcuse, One-dimensional man. Studies in the ideology of advanced industrial society, Boston 1964 und Helmut Schelsky, Der Mensch in der wissenschaftlichen Zivilisation [1961], in: Auf der Suche nach Wirklichkeit, hg. v. Helmut Schelsky, München 1979, 449-486.

${ }^{14}$ Niklas Luhmann, Ökologische Kommunikation. Kann die moderne Gesellschaft sich auf ökologische Gefährdungen einstellen?, Opladen 1990, $237 \mathrm{ff}$.

${ }^{15}$ Jan-Werner Müller, Furcht und Freiheit. Für einen anderen Liberalismus, Berlin 2019, hier 83 und 147, meint, in der liberalen Demokratie müssten zuerst die "Opfer" gehört werden. Allerdings dürfe deren Anspruch auf den Opferstatus nicht nur durch 
Die Kommunikation von Angst lässt sich leicht politisch instrumentalisieren. Sie ist »immer authentische Kommunikation, da man sich selbst bescheinigen kann, Angst zu haben, ohne dass andere dies widerlegen können «. ${ }^{16}$ Zwar kann die Behauptung, Angst zu verspüren nicht widerlegt werden, die Rationalität oder Triftigkeit von Angst aber schon. Dann liegt der Verdacht nahe, jemand spiele die Angst nur vor oder sei phobisch. Angstpolitik ist anfällig für den Ideologieverdacht.

In der englischsprachigen Literatur wird eine ideologische Angstpolitik als "politics of fear" bezeichnet. Es handelt sich um einen kritischen Begriff, der eine Art des Politikmachens markiert, in der Angst als ein illegitimes Mittel des Machterhalts und der Erreichung fragwürdiger Ziele genutzt wird. ${ }^{17}$ Der Begriff transportiert den Verdacht, mit Angst ließe sich ausschließlich auf eine Art und Weise Politik machen, die a priori kritikwürdig ist. In Wirklichkeit erwarten die Bürgerinnen und Bürger aber die Responsivität der Regierungen und Gesetzgeber gegenüber ihren Ängsten. Und dies wird von vielen Politikerinnen und Politikern, durch deren gebetsmühlenartig abgegebene Versprechen, "nah bei den Menschen« und ihren "Sorgen und Nöten" zu sein, auch anerkannt. Auf eine paradoxe Weise zeigt sich dies sogar anhand der oft zitierten Forderung nach "Freiheit von Furcht" ("freedom from fear"). Sie wurde, von Franklin D. Roosevelt über Willy Brandt bis hin zu Barack Obama, immer wieder von demokratischen Politikern als eine politische Parole genutzt. Abgesehen davon, dass sich eine vorgebliche Politik der Angstüberwindung bei genauerem Hinsehen als Angstpolitik erweisen könnte, werden in jedem Fall auch diejenigen Politikerinnen, die der Maxime »Freiheit von Furcht« folgen, nicht umhinkönnen, politische Entscheidungen dann und wann mit Blick auf tatsächliche oder vermeintliche Ängste der Bürgerinnen und Bürger zu treffen und zu rechtfertigen. Mit anderen Worten: In Demokratien ist Angstpolitik ein unvermeidbarer Teil des politischen Prozesses.

die Artikulation von Angst beglaubigt werden, er müsse sich in öffentlichen Debatten bewähren.

${ }^{16}$ Luhmann, Ökologische Kommunikation, 240.

${ }_{17}$ David L. Altheide, Terrorism and the politics of fear, Lanham 2006; Frank Furedi, The politics of fear. Beyond left and right, London 2005; Corey Robin, Fear. The history of a political idea, Oxford 2004. 


\section{Angstpolitik von links und rechts}

Idealtypisch lassen sich eine "rechte " und eine »linke« Angstpolitik unterscheiden. Historisch gehen sie auf die "Entstehung des Politischen bei den Griechen ${ }^{18}$ und deren Erfindung der Demokratie zurück. Bis heute sind sie eine Erscheinung der Politik in Demokratien und damals wie heute haben sie mit dem Konflikt zwischen "oben« und "unten« zu tun. ${ }^{19}$

Für die Aristokratie der antiken Polis stellte die Erfindung der Demokratie eine Bedrohung dar, denn die Idee der Demokratie versprach politische Gleichheit. Sie stellte damit Eigentum, Einfluss und Privilegien der aristokratischen Wenigen infrage. Die Mehrheit der einfachen Bürger hingegen hatte Angst vor den Herrschaftsansprüchen der Aristokraten. Die Vielen befürchteten die Umgehung, Schwächung oder Abschaffung der demokratischen Verfahren durch die Wenigen. In dieser Konstellation bildeten sich die Grundmotive der "rechten" und "linken" Angstpolitik heraus. Die Angst vor den demokratisch ermächtigten Vielen ist das Urbild der "rechten" Angstpolitik, die Angst vor den aristokratischen Wenigen ist die Grundfigur der »linken« Angstpolitik.

Man kann gegen diese Herleitung einwenden, dass es in die Irre des Anachronismus führe, wenn man neuzeitliche Kategorien wie "links" und "rechts" in die Antike zurückprojiziert, da die damaligen politischen Konflikte von den Zeitgenossen nicht mit diesen Kategorien beschrieben worden sind. Eine Deutung antiker Verhältnisse mit neuzeitlichen Kategorien verfehlt tatsächlich die Eigenheit der antiken Politikform. Gleichwohl stellt das, was hier unter "rechter" und »linker" Angstpolitik verstanden werden soll, eine je eigene Antwort auf eine politische Grundfrage dar, die nicht nur das antike politische Denken beschäftigt hatte: Wer ist mehr zu fürchten - die Vielen oder die Wenigen? Diese Frage ist so alt wie aktuell. Sie treibt die gegenwärtige Demokratietheorie um und sie gewinnt im zeitgenössischen politischen Denken durch den Aufstieg des Populismus an Relevanz. Ihre zeitgenössische Fassung lautet: Stellen die Eliten

\footnotetext{
${ }^{18}$ Christian Meier, Die Entstehung des Politischen bei den Griechen, Frankfurt am Main 1980.

${ }^{19}$ Vgl. Luciano Canfora, Eine kurze Geschichte der Demokratie. Von Athen bis zur Europäischen Union, Köln ${ }^{3} 2006$, 15-80; Moses I. Finley, Das politische Leben in der antiken Welt, München 1986; Ellen Meiksins Wood, Citizens to lords. A social history of Western political thought from Antiquity to the Middle Ages, London 2008, 28-98.
} 
oder die Bürger das größere Risiko für die liberale Demokratie dar? ${ }^{20}$

Von Platon über Edmund Burke und Madison bis zu zeitgenössischen Antidemokraten wie Jason Brennan ${ }^{21}$ besagt die "rechte" Antwort auf die Frage, wer mehr zu fürchten sei, dass vor allem die Vielen furchtbar seien, denn es mangele ihnen an Vernunft und Tugend. Politik solle das exklusive Betätigungsfeld einer weisen Elite sein, die dieVielen regiert (zu deren eigenem Besten). Werde die Politik der inkompetenten Masse und ihren demagogischen Anführern überlassen, führe das zu wirtschaftlichem Niedergang, kulturellem Verfall und politischem Aufruhr. In diesem geistigen Zusammenhang des antidemokratischen bis demokratieskeptischen Denkens stehen auch die Massentheorien des bürgerlichen Zeitalters, die in der Menge vor allem ein Ordnungsproblem sahen und Zivilisation zuvörderst mit der Disziplinierung der angeblich zügellosen Vielen verbanden..$^{22}$

Auch in der Linken gab es einen massenskeptischen Diskurs. Er drehte sich um die vermeintliche Führungsbedürftigkeit der Mas$\operatorname{sen}^{23}$ und zeigte Überschneidungen mit der "rechten" Denkweise. Dessen ungeachtet gibt es eine spezifisch »linke " Antwort auf die Frage, die sich von der "rechten « Antwort unterscheidet. Sie findet sich sowohl bei antiken Demokraten als auch bei Machiavelli wie bei neo-populistischen Demokraten wie John P. McCormick. ${ }^{24} \mathrm{Im}$ Kern besteht sie aus der dem massenskeptischen Denken entgegengesetzten These, dass in der Politik vor allem die Wenigen zu fürchten seien, denn diese seien aufgrund ihrer privilegierten Stellung an Bevorzugung gewöhnt und herrschsüchtig. In hohem Maße klassenund standesbewusst, erkannten sie die Bedrohung ihrerVorteile und Vorrechte durch demokratische Aspirationen. Infolge der ewigen

\footnotetext{
${ }^{20}$ Man kann sagen, dass diese Frage falsch gestellt ist, da sie von gesellschaftlichen Strukturen und politischen Institutionen absieht. Das ändert aber nichts an ihrer politischen Relevanz.

${ }^{21}$ Jason Brennan, Gegen Demokratie. Warum wir die Politik nicht den Unvernünftigen überlassen dürfen, Berlin 2017.

${ }^{22}$ Helmut König, Zivilisation und Leidenschaften. Die Masse im bürgerlichen Zeitalter, Reinbek bei Hamburg 1992; Joachim Schumacher, Die Angst vor dem Chaos. Gegenangriff durch Geschichte, zugleich Verteidigung der Demokratie, des Christentums, des Mutes des individuellen Mannes u. anderer missachteter Ideale, Paris 1937, Neuauflage Frankfurt am Main 1978.

${ }^{23}$ Siehe zur innerlinken Kritik einer Variante dieses Diskurses Hendrik Wallat, Staat oder Revolution. Aspekte und Probleme linker Bolschewismuskritik, Münster 2012. ${ }^{24}$ John P. McCormick, Niccoló Machiavelli, in: Radikale Demokratietheorie. Ein Handbuch, hg. v. Dagmar Comtesse u.a., Berlin 27-39.
} 
Schmeicheleien, die sie bekämen, und ihrer daraus resultierenden Selbstüberschätzung neigten sie zum Missbrauch von Macht. Lasse man sie gewähren, machten »die da oben« nur das, was ihnen selbst nützt.

Der Rechtspopulismus hat diese Trennung zwischen einer »linken" und einer "rechten" Angstpolitik durcheinandergebracht. Rechtspopulistinnen treiben eine Form von Angstpolitik, die zwischen links und rechts changiert. Das stellt eine politische Strategie dar, die Motive der »linken" Angstpolitik mit Motiven der "rechten" Angstpolitik zu verbinden. Die rechtspopulistische Antwort auf die Frage, wer ein Risiko für Freiheit und Demokratie ist, lautet: Die herrschende Elite und die fremden Massen. Antipopulisten aus allen politischen Lagern reagieren auf diese Strategie unter anderem mit einer Abwehr populistischer Impulse. Sie aktualisieren dabei Motive der Angst vor den unvernünftigen Vielen - mit der Folge, dass elitenkritische "populäre« Politik zum Alleinstellungsmerkmal der Rechtspopulisten wird.

\title{
Angstpolitik lechts und rinks
}

\author{
manche meinen \\ lechts und rinks \\ kann man nicht velwechsern \\ werch ein illtum!
}

Ernst Jandl, Lichtung

Populisten machen sich symbolisch zu den Fürsprechern des Volks und seines angeblichen Willens oder Wohls in Abgrenzung zu einer herrschenden, angeblich korrupten politischen Klasse oder Elite. Sie etablieren damit eine moralische Wir-Sie-Unterscheidung, in welcher die herrschende Minderheit als unmoralisch verdammt und das Volk als moralisch höherstehend glorifiziert wird. Dabei berufen sich Populistinnen auf demokratische Prinzipien wie die Volkssouveränität und die Responsivität der Regierenden. Das verweist darauf, dass es zwischen Demokratie und Populismus eine Verbindung gibt. Mit dem Politikwissenschaftler Michael Th. Greven kann man diese Verbindung im "populistischen Zug" der "Demokratie als Regierungspraxis" sehen:

"Schon immer und legitimerweise besaß die Demokratie als

Regierungspraxis einen populistischen Zug, der sich einerseits 
aus dem Wiederwahlwunsch der auf Zeit Regierenden ergab, und der andererseits über die Rückkopplung bei den seltenen Wahlen hinaus für ein gewisses $\mathrm{Maß}$ an Responsivität zu sorgen hatte. $\ll^{25}$

Früher haben die sogenannten »Volksparteien«, die mit dem Anspruch antraten, das gesamte Volk zu repräsentieren, sowie einige der sozialistischen und kommunistischen Parteien im Sinne dieses "populistischen Zugs" populistisch agiert. Allerdings trieben in ihrer Frühphase auch die GRÜNEN populistische Politik, als sie den "Altparteien« vorwarfen, abgehoben und undemokratisch zu sein.

Gegenwärtig wird der "populistische Zug« von der politischen Klasse nicht mehr sonderlich überzeugend auf die politische Bühne gebracht; einigen Bürgerinnen und Bürgern scheint er überhaupt nicht mehr vorhanden zu sein. Zudem haben sich viele linke Parteien und Bewegungen von der Mobilisierung mittels elitenkritischen Kollektivbegriffen verabschiedet und infolgedessen die Angst vor mächtigen Eliten nicht politisiert. Dies ermöglicht es populistischen Parteien, Bewegungen und Politikerinnen, sich in Abgrenzung zur politischen Klasse in Stellung zu bringen, die Angst vor übermächtigen Eliten zu artikulieren und im Namen von Volk und Demokratie aufzutreten. ${ }^{26}$ Es ist bemerkenswert, dass es vor allem dem Rechtspopulisten gelingt, diese Rolle für sich in Anspruch zu nehmen.

Rechtspopulistinnen fügen der populistischen Differenz zwischen korrupter Elite und gutem Volk eine weitere Unterscheidung hinzu. Sie unterscheiden zwischen dem guten Eigenen (dem eigenen Volk, der eigenen Nation, der eigenen Gemeinschaft) und dem bedrohlichen Fremden. In ihrer Mobilisierung konstruieren sie das "eigene Volk" als eine gute Gemeinschaft, die von zwei Seiten bedroht werde, von einer "korrupten Elite« und von "massenhaft einwandernden Fremden«. Damit verbindet der Rechtspopulismus in einer angstpolitischen Querfrontstrategie "rechte " mit »linken« Topoi. Die Angst vor einer herrschenden Minderheit verknüpft er mit der Angst vor einer gefährlichen Masse Fremder (die durch die herrschende Minderheit ins Land gelassen werde). Dabei sind auch interessen- und Identitätspolitische Topoi von Bedeutung, denn

\footnotetext{
${ }^{25}$ Michael Th. Greven, Kampagnenpolitik, in: Kontingenz und Dezision. Beiträge zur Analyse der politischen Gesellschaft, hg. v. Michael Th. Greven, Opladen 2000, 137-152, hier 130.

${ }^{26}$ Ausführlich Dirk Jörke/Veith Selk, Theorien des Populismus zur Einführung, Hamburg 2017; Veith Selk, Revolte von rechts. Der Aufstieg des Retropopulismus als Reaktion auf gegenwärtige Demokratieprobleme, in: Fromm Forum 24 (2020), 124-149.
} 
die rechtspopulistische Mobilisierungsstrategie basiert auch auf der Behauptung, sowohl die Interessen als auch die Identität "des Volks" würden von der Elite und den Fremden bedroht. Diese Strategie entspricht der Stimmungslage. Die jährliche Umfrage der R+V Versicherung nach »Den größten Ängsten der Deutschen« listet für das Jahr 2019 auf Platz eins die Angst vor »Überforderung des Staats durch Flüchtlinge«. Die Angst vor "Spannungen durch Zuzug von Ausländern« folgt auf dem zweiten Platz. Auf dem vierten Platz liegt die Angst vor der »Überforderung der Politiker ${ }^{27}$

Die Antipopulisten aus allen Lagern reagieren auf diese Herausforderung mit einem Programm, das man als postdemokratischen Liberalismus bezeichnen kann. ${ }^{28}$ Der postdemokratische Liberalismus nimmt Abschied von der Idee von Demokratie als derVerwirklichung eines demokratisch gebildeten Mehrheitswillens. Er setzt stattdessen auf ein gutes Regieren im Dienste liberaler Werte wie privater Freiheit und Wachstum. Es solle vor allem durch Expertenwissen geleitet werden, könne aber auch durch die Beteiligung gut informierter Bürgerinnen und Bürger, "im Dialog«, unterstützt werden.

In seiner "linken "Variante hebt der postdemokratische Liberalismus den Wert der Chancengerechtigkeit stärker hervor; in der "rechten« Variante sitzt sein Akzent eher auf individueller Leistungsgerechtigkeit. Beide Spielarten des postdemokratischen Liberalismus teilen jedoch die Stoßrichtung der wettbewerblichen Anpassung an den globalen Kapitalismus und die vermeintlichen "Sachzwänge" der Marktgesellschaft, die sie als im Ganzen nicht steuer- und beherrschbar auffassen. Öffnungsprozesse in der Politik (Europäisierung, Globalisierung), in der kapitalistischen Ökonomie (Freihandel, Flexibilisierung und Innovation) und in der Kultur (Toleranz und Vielfalt) werden in dieser Denkweise als miteinander zusammenhängende Mittel der Anpassung im "globalen Wettbewerb um Märkte, Köpfe und Kapital« begriffen.

Postdemokratische Liberale beschreiben den Rechtspopulismus als eine Gefahr für die liberale Regierungsweise und dessen Werte. Von ihm seien sowohl Minderheiten als auch das Große und Ganze des liberaldemokratischen Kapitalismus bedroht. Sein unvernünftiges protektionistisches Programm und dessen Verkennung von Diversität und Offenheit als wichtigen Standortfaktoren bedrohten

\footnotetext{
${ }^{27}$ https://www.ruv.de/static-files/ruvde/Content/presse/die-aengste-der-deutschen/aengste-grafiken/ruv-aengste-grafiken.pdf (20.12.2019). Auf Platz drei liegt die Angst vor einer "gefährlicheren Welt durch Trump-Politik".

${ }^{28}$ Dirk Jörke/Veith Selk, Was ist postdemokratischer Liberalismus?, in: Journal für politische Bildung 1 (2019), 24-27.
} 
den Wohlstand. Die illiberale Politik des Rechtspopulismus ziele auf »bloße« Mehrheiten.

\section{Schluss: Keine Demokratie ohne Mehrheitspolitik}

Rechtspopulistische Angstpolitik geht auf Kosten von schwachen Minderheiten, deshalb ist die antipopulistische Kritik an ihr in dieser Hinsicht berechtigt. Jedoch ist die Strategie des postdemokratischen Liberalismus, Mehrheitspolitik als prinzipiell gefährlich darzustellen und in der Auseinandersetzung mit der rechtspopulistischen Herausforderung auf eine anti-majoritäre Angstpolitik zu setzen, riskant. Auf lange Sicht stellt der angstpolitische Anti-Majoritarismus eine unkluge Strategie dar, denn sowohl der Anspruch auf eine demokratische Politik für die Mehrheit als auch die Repräsentation der Angst vor starken Minderheiten wird hierdurch zu einem Alleinstellungsmerkmal rechtspopulistischer Parteien. Und diese wissen ihre Chance zu nutzen. Zur Demokratie gehören schließlich, wie Margaret Canovan gezeigt hat, ${ }^{29}$ nicht nur die Erwartung guten Regierens seitens professioneller Politikerinnen und Politiker, sondern auch das Versprechen, mittels Stimmzetteln mächtige Minderheiten in ihre Schranken zu weisen und die Lebensverhältnisse der Mehrheit zu verbessern.

- Dr. Veith Selk arbeitet als wissenschaftlicher Mitarbeiter am Arbeitsbereich Politische Theorie und Ideengeschichte des Instituts für Politikwissenschaft der TU Darmstadt. Zu seinen Schwerpunkten gehören unter anderem Demokratie, Populismus, Angst \& Politik.

${ }^{29}$ Margaret Canovan, "Trust the people!« Populism and the two faces of democracy, in: Political Studies 47 (1999), 2-16. 